Bioscientia Medicina: Journal of Biomedicine \&

Translational Research

Journal Homepage: www.bioscmed.com

\title{
Programmed Death-Ligand 1 Protein and Colorectal Cancer Patient Survival Rates in Hasan Sadikin Hospital Bandung
} Vandra Bina Riyanda ${ }^{*}$, Reno Rudiman ${ }^{2}$, Nurhayat Usman ${ }^{2}$

1 Trainee Digestive Surgeon Department, Faculty of Medicine, Padjajaran University, Bandung, Indonesia

2 Digestive Surgeon Department, Faculty of Medicine, Padjajaran University, Bandung, Indonesia

\section{A R T I C L E I N F O}

Keywords:

PD-L1

Colorectal cancer

Survival rate

*Corresponding author:

Vandra Bina Riyanda

E-mail address:

\section{vandrariyanda@gmail.com}

All authors have reviewed and approved the final version of the manuscript.

\begin{abstract}
A B $\mathbf{S}$ T $\mathbf{R}$ A $\mathbf{C}$ T
Background: According to the American Cancer Society, Colorectal Cancer (CRC) is the third leading cause of cancer death in men and women in the United States. In Indonesia, CRC ranks as the third most common malignancy in both men and women. Programmed death-ligand 1 (PD-L1) is a trans-membrane receptor ligand and negative regulatory signal for $\mathrm{T}$ cells that is elevated in several tumors including $\mathrm{CRC}$ and binds to programmed death 1 (PD-1) on T cells, B cells, dendritic cells and natural killer T cells. PD-L1 expression was found in tumor cells and tumor cells that infiltrate immune cells in several malignancies, including CRC. Methods: This study is a comparative analytic cross sectional study with consecutive sampling method. This study was conducted at Hasan Sadikin Hospital, Bandung from September to October 2021. Further statistical analysis was done SPSS version 25.0 for Windows (SPSS Inc., Chicago, Ill., USA). Results: The total sample in this study was 50 subjects with colorectal cancer patients at RSHS. The number of CRC patients who expressed PDL1 were $23(46 \%)$ and 27 subjects (54\%). The majority of cancer patient survival 2 Years was 38 subjects $(76.0 \%)$ and survivors of more than 2 years was 12 subjects $(24.0 \%)$. Conclusion: There is no significant relationship between PD-L1 and survival rates in CRC patients.
\end{abstract}

https://doi.org/10.37275/bsm.v6i1.437

\section{Introduction}

Based on the Colorectal Cancer (CRC) Management Guidelines of the Ministry of Health of the Republic of Indonesia in $2015, \mathrm{CRC}$ is a malignancy originating from colon, consisting of the colon (the longest part of the colon) and/or the rectum (the last part of the colon before the anus).1,2 CRC is a malignancy in the colon or rectum that originates in the epithelial. This cancer generally affects elderly humans and is very rare in children. Carcinoma can be diagnosed through definitive biopsy, including fine needle aspiration (FNA), core biopsy, or subtotal removal of a single node. Microscopic examination by a pathologist is then required to identify the molecular, cellular or tissue characteristics of epithelial architecture. ${ }^{3}$

Based on the GLOBOCAN survey (Global Burden of Cancer Study) 2018, worldwide incidence ranks third, namely 1360 of 100,000 population [9.7\%] male and female and was ranked fourth as the cause of death that is 694 out of 100,000 population [8.5\%], male and female. According to the American Cancer Society, the number of new cases in the United States in 2016 was about 95,270 colon cancer patients and 39,220 rectal cancer patients. According to the American Cancer Society, CRC is cancer and is the third leading cause of cancer death in men and women in the United States. 1

In Indonesia, CRC ranks as the third most common 
malignancy in both men and women after lung cancer and breast cancer with a percentage of $21.0 \%$ in men and $14.0 \%$ in women from the total number of cancer patients in Indonesia. The ratio of incidence in men and women is 3 to 1 and less than 50\% of colon and rectal cancers are found in the rectosigmoid.1 Other data from the Ministry of Health shows the incidence of CRC with age less than 45 years in 4 major cities in Indonesia as follows, Jakarta 47, 85\%, Bandung 54.5\%, Makassar 44.3\% and Padang 48.2\%. ${ }^{1}$

Diabetic foot ulcers that are chronic and difficult to heal are the most common cause of non-traumatic amputation (lower leg amputation (ALL) in DM patients, reaching $82 \%$. Observational research on $94 \mathrm{DM}$ patients at Dr. Kariadi Hospital Semarang found that the incidence of lower extremity amputation increased in diabetic foot ulcers by $31.9 \%$. lower extremity. ${ }^{4}$

The exact cause of CRC is unknown. Some experts can't explain why people have CRC. Generally, CRC is a result of an interaction between environmental factors and genetic factors. Some of the risk factors are age, heredity, physical activity, diet and nutrition patterns, consumption of cigarettes and alcohol.4,5,6,7 Signs and symptoms include bleeding in the lower gastrointestinal tract which is characterized by bloody discharge during defecation, an increase in the amount of stool produced and often accompanied by diarrhea that lasts for more than 6 weeks. In addition, other symptoms include signs of intestinal obstruction and weight loss.

There are many immunohistochemical (IHC) biomarkers in CRC such as calretinin, MUC2, COX-2, Cadherin-17, EGFR, VEGF, etc. IHC is generally used to identify and classify a carcinoma. However, apart from identifying and classifying carcinomas, IHC also plays an important role in the selection of therapies, such as PD-L1 which can be used for cancer treatment with immunotherapy.

Programmed death-ligand $1(\mathrm{PD}-\mathrm{L} 1)$ is a transmembrane receptor ligand and negative regulatory signal for $\mathrm{T}$ cells that is elevated in several tumors including $\mathrm{CRC}$ and binds to programmed death 1 (PD1) on T cells, B cells, dendritic cells and natural killer $T$ cells. making tumor cells have a mechanism to be able to avoid immune attacks until the suppression of immune cells.8,9Many previous reports have suggested that high PD-L1 expression in tumor cells is correlated with poor clinical progress in other tumor types, such as malignant melanoma, non-small cell lung cancer and CRC. The poor prognosis in patients with high PDL1 expression is thought to be due to the activity of the PD-1/PD-L1 pathway, which induces T-cell immune suppression in the tumor microenvironment. However, the underlying mechanism remains unclear. To clarify the biological implications of PD-L1 activity, several studies have shown that PD-L1 binding to PD-1 negatively regulates $\mathrm{T}$ cell synthesis of interleukin 2 and interferon-gamma, resulting in apoptosis of CD8+ cytotoxic $\mathrm{T}$ lymphocytes in vitro. Further biological elucidation of the role of $\mathrm{PD}-\mathrm{L} 1$, including in vivo experiments. 10

Immunotherapy is developing rapidly and there are still many undiscovered facts regarding the level of PDL1 expression in individual tumor tissues that play a role in identifying patients most likely to respond to immunotherapy. For example, in Hodgkin's lymphoma, most tumors express very little PD-L1, so immunotherapy is not appropriate for this tumor type. However, for certain cancer groups, evidence showed that the response to PD-1 inhibitors (pembrolizumab and nivolumab) or anti-PD-L1 antibodies (atezolizumab and durvalumab) was highly responsive. Therefore, assays detecting PD-L1 expression play an important role in the use and development of anti-PD-1/PD-L1 drugs aimed at this tumor type, including bladder/urothelial cell, lung, gastric, colon and colorectal cancers. The PD-L1 protein test is usually designated on antibody clones used to detect the presence of the PD-L1 protein. The 22C3 assay developed by Dako (Pharm Dx PD-L1 IHC 22C3. Agilent Pathology Solutions) uses a mouse monoclonal antiPD-L1 clone. Several available assays have been developed and validated as part of a clinical trial used to demonstrate the efficacy of PD-1/PD-L1 immunotherapy drugs.

Several studies of molecular markers such as BRAF, KRAS and MMR play a role in predicting recurrence in stage III CRC. In addition, the use of PD-1 and PD-L1 has now been developed as a pathway for providing negative feedback on the activity of $\mathrm{T}$ cells. PD-L1 
expression was found in tumor cells and tumor cells that infiltrate immune cells in several malignancies, including CRC. In addition, it is currently suspected that the expression of PD-L1 on tumor cells and tumor infiltrating mononuclear cell (TIMC) is associated with the prognosis of various tumors. The prognostic relationship between PD-L1 expression and CRC cancer is still not widely studied.9.10

This study is based on the results of previous epidemiological studies, it is known that the incidence of CRC is very high, even Bandung is the fourth city with the highest incidence rate in Indonesia. Based on medical record data at Hasan Sadikin Hospital, CRC is the third most common cancer. Hasan Sadikin Hospital itself, as a level 3 health facility accepts and treats CRC patients who are referred from level one or level two health facilities. In addition, there are several factors that affect the survival rate of $\mathrm{CRC}$ patients. In general, the overall survival of patients diagnosed with stage III $\mathrm{CRC}$ is around $74.6 \%$ in the first year, 43.8 at 5 years and $33 \%$ at 10 years. Based on these results of this study are expected to give more insight, and better data or to be used in further research.

\section{Methods}

This study is a comparative analytic cross sectional study. The sampling method used in this study was consecutive sampling at Hasan Sadikin Hospital, Bandung from September to October 2021. The population of this study were all patients who had been diagnosed with CRC at Hasan Sadikin Hospital, Bandung. The subjects were sent for histopathological examination and further management at the Digestive Surgery outpatient department, Hasan Sadikin Hospital, Bandung. The inclusion criteria in this study were Patients diagnosed with CRC which has been examined histopathologically and consented to participate in this study. The exclusion criteria for this study was patient with comorbid diseases (diabetes mellitus, hypertension, and thyroid disorders, and heart disease). This research has been approved by Hasan Sadikin Hospital Ethical Committee.

Data collection from patients was carried out in the digestive surgery outpatient department and histopathological data at the anatomical pathology laboratory at Hasan Sadikin Hospital, Bandung. The data collected were age, gender, location of cancer, CRC stage, PD-L1 expression level and survival rate. Statistical analysis was done using Saphiro-Wilk test for data normality test, Kolgomorov Smirnov test, Mann-Whitney test, chi square test, unpaired t-test. Software used was SPSS version 25.0 for Windows (SPSS Inc., Chicago, Ill., USA).

\section{Results}

The total sample in this study was 50 people with colorectal cancer patients at RSHS. Characteristics of patients when viewed by age in table 1 shows that the majority age is in the range of 50-60 years with a percentage of $30.0 \%$. Then the second highest ranking is those aged between 40-50 years, amounting to 11 people or $20 \%$. When viewed by gender, the majority of cancer patients were women, amounting to 42 people $(42.0 \%)$. Based on the table, it can be seen that the patient's PDL-1 protein was divided into 23 people $(46.0 \%)$ having positive results and 27 people $(54.0 \%)$ having negative results.

Majority of cancer patient survival 2 years which amounted to 38 people $(76.0 \%)$ then the survivors who were more than 2 years were 12 people $(24.0 \%)$. It is known that most of the cancers in the patients were in the stage III category, amounting to 29 people $(58.0 \%)$. 
Table 1. Patient characteristics data $(n=50)$

\begin{tabular}{|c|c|c|c|}
\hline No & Characteristics & Amount & Percentage (\%) \\
\hline \multirow[t]{7}{*}{1} & Age & & \\
\hline & 20 Years & 2 & $4.0 \%$ \\
\hline & 21-30 Years & 8 & $16.0 \%$ \\
\hline & $31-40$ Years & 8 & $16.0 \%$ \\
\hline & 40-50 Years & 11 & $22.0 \%$ \\
\hline & 51-60 Years & 15 & $30.0 \%$ \\
\hline & $>60$ Years & 10 & $20.0 \%$ \\
\hline \multirow[t]{3}{*}{2} & Gender & & \\
\hline & Man & 29 & $58.0 \%$ \\
\hline & Woman & 42 & $42.0 \%$ \\
\hline \multirow[t]{3}{*}{3} & PD-L1. protein & & \\
\hline & Positive & 23 & $46.0 \%$ \\
\hline & Negative & 27 & $54.0 \%$ \\
\hline \multirow[t]{3}{*}{4} & Survival & & \\
\hline & 2 Years & 38 & $76.0 \%$ \\
\hline & $>2$ Years & 12 & $24.0 \%$ \\
\hline \multirow[t]{5}{*}{5} & Stadium & & \\
\hline & $\overline{\mathrm{I}}$ & 0 & $0.0 \%$ \\
\hline & II & 12 & $24.0 \%$ \\
\hline & III & 29 & $58.0 \%$ \\
\hline & IV & 9 & $18.0 \%$ \\
\hline
\end{tabular}

Table 2. Hypothesis Testing $(n=50)$

\begin{tabular}{|c|c|c|c|c|c|c|c|}
\hline \multirow[t]{2}{*}{ Protein PDL-1 } & & \multicolumn{2}{|c|}{ Onset } & \multirow[t]{2}{*}{ Total } & \multirow[t]{2}{*}{ p-value } & \multirow[t]{2}{*}{$\mathrm{CC}$} & \multirow[t]{2}{*}{ OR } \\
\hline & & $<=2$ years & $>2$ years & & & & \\
\hline \multirow[t]{2}{*}{ Positive } & $\mathrm{n}$ & 16 & 7 & 23 & 0.325 & 0.138 & 0.519 \\
\hline & $\%$ & $69.6 \%$ & $30.4 \%$ & $100 \%$ & & & \\
\hline \multirow[t]{2}{*}{ Negative } & $\mathrm{n}$ & 22 & 5 & 27 & & & \\
\hline & $\%$ & $81.5 \%$ & $18.5 \%$ & $100 \%$ & & & \\
\hline \multirow[t]{2}{*}{ Total } & $\mathrm{n}$ & 38 & 12 & 50 & & & \\
\hline & $\%$ & $76 \%$ & $24 \%$ & $100 \%$ & & & \\
\hline
\end{tabular}

\section{Discussion}

$\mathrm{CRC}$ is a malignancy originating from intestinal tissue large, consisting of the colon (the longest part of the large intestine) and/or rectum (last small part of the large intestine before the anus).1,3 PD-L1 is a trans-membrane receptor ligand and negative regulatory signal for $\mathrm{T}$ cells that is elevated in several tumors including colorectal and binds to Programmed death 1 (PD-
1) in T cells, B cells, dendritic cells and natural killer T cells, make tumor cells have a mechanism to be able to avoid immune attacks until there is suppression of immune cells. 8,9

In recent years, immunotherapy has developed in the management of cancer, especially in cases with PD-L1. PD-L1 is an important stimulatory signal, which can cause suppression of the immune system, the PD-L1 
pathway becomes a promising prospect for immunotherapy in several cancers such as melanoma, non-small cell lung cancer, head and neck cancer, malignant lymphoma and urothelial cancer. However, the prognostic features of CRC with PD-L1 have inconsistent results. In many studies it has been stated that CRC with PD-L1 expression has a poor prognosis, but in some studies the results are not significant. 11

This study was conducted to observe the relationship between $\mathrm{CRC}$ and $\mathrm{PD}-\mathrm{L} 1$ on patient survival. Many studies have been conducted to assess the relationship between CRC survival and PD-L1 expression. Hamada et al stated that there was a poor prognostic relationship in CRC patients who expressed PD-L1. Song et al also stated that there was an association with poor prognosis in PD-L1-expressed CRC patients. ${ }^{11}$

In our study, research samples were collected from medical record data of CRC patients at RSHS from 2016-2018. In this study sample, 23 patients (46\%) expressed PD-L1 and 27 patients (54\%) did not express PD-L1. In the study of Thao Shan et al, there were $57 \%$ of patients who expressed PD-L1, so that when compared with this study, the number of patients who expressed PDLI was almost the same. In this study, the results that the $\mathrm{PD}-\mathrm{L} 1$ protein did not have a significant relationship with survival in cancer patients $(p>0.005)$.

If we see research results that are inconsistent with the results of previous studies, it is very possible because this study did not assess the DNA of CRC. In this study also obtained the number of samples with advanced stages of 38 people (76\%) while the research conducted by Tao Shan et al totaled 54 people $(67 \%)$. When viewed from the percentage of the number of patients in this study, the number of patients with advanced stages was more than the research conducted by Tao Shan et al, thus providing different research results from previous studies. As we know that advanced stages of CRC patients will give a poor survival rate regardless of PD-L1 expression. ${ }^{12}$

According to Droeser et al. CRC patients with DNA mismatch repair (MMR) status with high PDL1 expression were associated with a good prognosis in CRC patients. PD-L1 expression was also associated with early tumor stage, low tumor grade, no lymph node metastases and no vascular invasion. In this study also explained that PD-L1 also has a relationship with IFN- $\gamma$ associated with a good prognosis because of IFN$\gamma$ stimulate tumor infiltration by $\mathrm{T}$ cells. ${ }^{13}$

PD-L1 expression in CRC patients is directly proportional to the amount of tumor infiltrating CD8 T cells. These cells do not express PD-1 so they are not affected by PD-L1 and infiltrate tumor cells, thereby interfering with tumor cell growth. In CRC patients with MMR there is an increased infiltration of CD8. T cells on tumor cells thus providing a good prognosis in CRC patients. 13

Increased IFN- $\gamma$ CRC tumor cells are also associated with a good prognosis. These cytokines are produced by activated $\mathrm{T}$ cells to promote PD 1 in tumor cells. Thus, patients who express high PD-L1 display elevated IFN- $\gamma$ which is activated by $T$ cells that infiltrate tumor cells so that it interferes with tumor cell growth. 13

Yaqi Li et al explained that PD-L1 expression in CRC was associated with a good prognosis assessed for OS and DFS especially in MMRproficient patients. However, several studies have shown that PDL-I expression has a poor prognosis in renal cell carcinoma, breast cancer, small lung cancer and osteosarcoma. Sabatier et al stated that PD-L1 expression in breast cancer was associated with a good prognosis for metastases free survival, OS and chemotherapy response. Darb-Esfahani et al also stated that the 
expression of PD-L1 gives a good prognosis in ovarian cancer patients. The good prognosis in PD-L1 overexpressing tumors may be the result of upregulation of the immune system that compensates for PD-L1 expression. ${ }^{14}$

However, there are also some studies that are not in accordance with this study. Tao Shan et al stated that PD-L1 is a very important molecule in tumor microenvironment. PD-L1 is a protein that plays an important role in the apoptosis of the immune response. After binding to the PD 1 receptor on cells, PD-L1 can induce apoptosis in the $\mathrm{T}$ set. Expression of PD-L1 in CRC has no significant relationship with tumor size, age, sex, and metastases. PD-L1 is an independent factor in the poor prognosis of CRC, but PD-L1 is associated with poor prognosis is still controversial. 12

Lianzhou et al also stated that the results were inconsistent with this study, that the expression of $\mathrm{PD}-\mathrm{L} 1$ in $\mathrm{CRC}$ indicates a poor prognosis in CRC patients. PD-L1 causes T cell infiltration in tumors to be poor so that the function of T cells to eliminate tumors is reduced. PD-L1 was not associated with age, gender, tumor location, metastases and MSI/MMR status. 11

So it can be concluded that the relationship of PD-L1 with CRC patient survival is still controversial. There are many studies that state that PD-L1 is associated with poor prognosis in CRC patients. However, there are also several studies which state that PD-L1 is associated with a good prognosis in CRC. In this study, there was no significant relationship between PD-L1 and poor prognosis in CRC patients. Further research is needed to assess the relationship of PD-L1 with the survival of CRC patients because many other factors affect the prognosis.

In this study, there are still some shortcomings. First, the number of samples used is still relatively small. In this study, researchers collected a sample of 50 patients. As we know, the more research we observe, the more representative the research results will be. The two expressions of PD-L1 assessed by antibody and dilution were different and would affect the sensitivity of the immunohistochemistry. The three MSI/MMR statuses in these patients were not examined. As previously explained, there is a relationship between MSI/MMR status and PDL1 expression. However, overall the results of this study can show that there is no significant relationship between $\mathrm{PD}-\mathrm{L} 1$ and the survival rate of CRC patients.

\section{Conclusion}

There is no significant relationship between PD-L1 and survival rates in CRC patients.

\section{References}

1. Colorectal cancer management guide. National cancer prevention committee. 2015

2. Siegel RL, Miller KD, Jemal A. Cancer statistics, 2016. 2016; 66: 7-30.

3. Amanda C.J. Faecal immunochemical tests (FIT) versus colonoscopy for surveillance after screening and polypectomy: a diagnostic accuracy and cost-effectiveness study. Gut. 2019; 68(9): 1642-1652.

4. Wolf A.M et al. Colorectal cancer screening for average-risk adults: 2018 guideline update from the American Cancer Society. CA: a cancer journal for clinicians. 2018; 68(4): 250-281.

5. Enriqueet all. Colonoscopy versus fecal immunochemical testing in colorectalcancer screening. New England Journal of Medicine. 2012; 366(8): 697-706.

6. Keith L M, Arthur F D, Anne M.R. 
Clinically oriented anatomy. Clinically

7. Pandey M, Swain J, Iyer H M., Shukla, M.

Primary lymphoma of the colon: Report of two cases and review of literature. World journal of surgical oncology. 2019: 17(1); $1-7$.

8. Hall, J. E., \& Hall, M. E. Guyton and Hall textbook of medical physiology e-Book. Elsevier Health Sciences. 2020

9. Palucka, A. K., and Coussens, L. M. The Basis of Oncoimmunology.

Cell 164. 2016; 1233-1247

10. Zhang Nengpan, et.al. 2017 Peptide Blocking of PD-1PD-L1 Interaction for Cancer Immunotherapy, 2017, DOI: 10.1158/2326-6066. CIR-17-0035

11. Prognostic and clinicopathological value of PD-L1 in colorectal cancer: a systematic review and meta-analysis PubMed [Internet]. [cited 2021 Nov 2]. Available oriented anatomy. 2018

https://pubmed.ncbi.nlm.nih.gov/3119 0869/

12. Tao Shan. PD-L1 Expression in Colon Cancer and its Relationship with Clinical Prognosis. Int J Clin Exp Pathol 2019; 12(5): 1764-1769

13. Clinical impact of programmed cell death ligand 1 expression in colorectal cancer ScienceDirect [Internet]. [cited 2021 Nov 2]. Available from: https://www.sciencedirect.com/science /article/abs/pii/S0959804913001238

14. Prognostic impact of programed cell death-1 (PD-1) and PD-ligand 1 (PD-L1) expression in cancer cells and tumor infiltrating lymphocytes in colorectal cancer - PubMed [Internet]. [cited 2021 Nov 2]. Available from: https://pubmed.ncbi.nlm.nih.gov/2755 2968/ 